\title{
COMMENT Technoference over time and parenting
}

\author{
Nerissa S. Bauer ${ }^{1}$ \\ Pediatric Research (2018) 84:157-158; https://doi.org/10.1038/s41390-018-0059-z
}

According to the Pew Research Center, the access to and use of smartphones and other mobile devices (e.g., tablets) has rapidly increased, such that $84 \%$ of American households have at least one smartphone and a third of all households had three or more. Coupled with the availability of broadband Internet, more smartphone owners are using mobile devices as their primary way to get connected. These devices have drastically changed how our society connects with each other socially online, but also increased the use of taking and sharing photos, streaming movies, catching up on news events, or how we shop and bank. It has also ushered in an abundance of apps for children and teenagers. There are apps to help children practice math facts, learn foreign languages, build imaginary worlds and learn coding. When it comes to raising children, it has rapidly added another dimension to parenting in the digital age such that parents must grapple with how to monitor their children's own use at home and school and promote safe digital citizenship, even if their own skills in using technology are limited.

In their study, "Technoference: Longitudinal Associations Between Technology Use, Parenting Stress, and Child Behavior Problems," McDaniel and Radesky, examined the complex interplay between parental use of technology during parent-child interactions and child behavioral outcomes. The authors are to be commended for their research, extending their prior work and utilizing a prospective dataset to examine outcomes across three time points. The sample accounted for maternal and paternal report on "technoference" or the degree to which technology intruded upon mother-child or father-child activities during a typical day. The authors noted that their sample was comprised of a large majority of Caucasian, cohabiting, and fairly educated families and thus findings may not be generalizable to the US population at large. However, this study adds to the growing scientific evidence examining parental use of technology, the role of parental stress, parent-child interactions and child behaviors. The authors found that parents who used their smartphones during parent-child interactions reported more difficulties with their child's behavior. Moreover, when children had difficult and challenging behaviors, it created more parental stress, which caused parents to withdraw from their children even more. This pattern was also true for children with internalizing behaviors; however, mostly pronounced for children who exhibited more withdrawal behaviors. The child who tends to "prefer to be alone" may mislead parents into thinking the child is otherwise fine and does not need parental support or attention, freeing up parents to use their own smartphones or attend to other tasks. The "technoference" of technology is complicated and transactional, but important to understand when children present with either externalizing or internalizing behaviors.
It was somewhat surprising that parental depressive symptoms were not a significant factor given known associations between parental depression and parenting practices. However, this may be related to the make up of the sample as effects for this association is strongest for studies among women of lower socioeconomic status and among mothers of infants. ${ }^{1}$ In future prospective studies on this topic, it may be helpful to examine whether there is a differential effect based on whether parents report current or past depressive symptoms. The authors' ability to demonstrate the mediating role parenting stress has on parental technology use and problematic child behavior may have clinical practice implications when replicated in future studies with more diverse samples.

Pediatric providers should be mindful of monitoring parents' use of smartphones and how it impacts observable parent-child interactions in the clinic. Pediatricians may want to inquire about parenting stress should parents raise concern about their child's behavior. They can also ask about coping mechanisms parents employ to handle their child's behavior or to manage their own stress, including asking about each family members' use of technology.

Other places children encounter technology is in schools. Given that schools incorporate technology in teaching children as young as Kindergarten, it is important to acknowledge the benefits technology has on how educators can incorporate it into the classroom and providing different exciting avenues how our children learn and take in the world. However, as with anything, moderation is important given that excess time on screens may lead to less time for physical activity and diminished opportunities for meaningful person-to-person conversation with others at school, at the playground and at home.

Understanding how family members use technology in the home is important. Asking how much screen time children get on smartphones, tablets, televisions or computers during the week and on weekends and whether parents keep devices charging outside the child's room overnight are essential to determining whether technology use is impairing sleep routines or sleep quality. Moreover, while parents acknowledge the use of technology to manage child behavior, feelings of tension and guilt are not uncommon and provides a valuable opportunity for pediatricians and other primary care providers to discuss these concerns as it relates to promotion of optimal child development, behavior and health.

There are two essential parenting principles to promote and share with parents early and often: the value of parental attention and the power of parental role modeling appropriate and acceptable behaviors. Parents are their children's first teachers modeling social interactions during every day interactions. As such, they play a pivotal role in their child's social-emotional

\footnotetext{
${ }^{1}$ Indiana University School of Medicine, Indianapolis, IN, USA
}

Correspondence: Nerissa S. Bauer (nsbauer@iupui.edu)

Received: 21 April 2018 Accepted: 1 May 2018

Published online: 13 June 2018 
development. Pediatricians can highlight this point at every well child visit, drawing parents' attention to their children's cues. The infant's first social smile is a milestone that parents can truly "see" the impact of their response to their infant's bids for attention and how their reciprocal response to their infant makes for a positive and engaging social interaction. These "serve and return" interactions are fundamental to the wiring of the brain around communication and social skills. Occasional inattention, in the setting of otherwise timely and responsive parental attention and love, is likely to not be a cause of overall concern. However, when limited or absent, it impacts the developing brain architecture and sets children up for poorer outcomes related to learning, behavior and health in later life. ${ }^{2}$

Parental attention and role modeling are also critical to a child's vocabulary and language development, which is correlated strongly to overall childhood cognitive development. The landmark study by Hart and Risley highlighted the profound impact daily verbal exchanges in the home contributes to children's language and vocabulary development. ${ }^{3}$ Moreover, a toddler's vocabulary at 24 months of age is a strong predictor of their ability to self-regulate their emotions. ${ }^{4}$ In the discussion of plausible mechanisms, McDaniel and Radesky point to the possibility that mobile device use displaces important verbal and nonverbal interaction and parental responsiveness over time, thus diminishing parents' opportunities to provide scaffolding to shape positive ways for children to learn how to regulate their behavior. Parents can be taught to coach toddlers on how to recognize and label both positive and negative emotions and model acceptable ways to manage these feelings and ask for help. When parents provide this positive, social-emotional scaffolding it promotes self-regulation skills and positive parent-child interactions. If parents are unable to attend to their child's needs, either due to "technoference" or not knowing how to intervene with a child when emotionally dysregulated, the teachable moment is lost. Thus, pediatricians can help parents understand the need to respond to their child's cues, talk to and with them and how to engage with their children in positive ways that promote child self-esteem and optimize their social-emotional development.

As the number of apps continues to rise, newer and faster smartphones and other technologies are developed and access to technologies for more families continues, it becomes increasingly important for ongoing rigorous research that includes objective and prospective data. However, this study reaffirms the fact that we can no longer neglect talking about family use of technology during well child visits. The American Academy of Pediatrics has published guidelines for pediatricians to address both traditional and newer technologies with families, as well as encouraging families to develop a family media plan. ${ }^{5}$ The guidelines recognize the educational value of such technologies but also emphasizes the importance of shared parent-child media use and ensuring use does not prevent children from participating in other non-screen activities essential to overall health and development. Helping families be mindful about everyone's use of technology, establishing clear family rules to prevent overuse and building in screen-free time to ensure quality inperson, synchronous face time with each other. Pediatricians can also educate parents about useful websites, such as Common Sense Media (www.commonsensemedia.org), and advise parents to explore apps to familiarize themselves with how it works and content are additional ways to keep parents abreast of the ever-changing digital landscape.

\section{ADDITIONAL INFORMATION}

Competing interests: The authors declare no competing interests.

Publisher's note: Springer Nature remains neutral with regard to jurisdictional claims in published maps and institutional affiliations.

\section{REFERENCES}

1. Lovejoy, M. C., Graczyk, P. A., O'Hare, E. \& Neuman, G. Maternal depression and parenting behavior: a meta-analytic review. Clin. Psychol. Rev. 20, 561-592 (2000).

2. The Science of Neglect: The Persistent Absence of Responsive Care Disrupts the Developing Brain. Working Paper No. 12. (Center on the Developing Child, Harvard University, 2012).

3. Hart, B. \& Risley, T. R. Meaningful Differences in the Everyday Experience of Young American Children. (Paul H Brookes Publishing, Baltimore, MD, 1995).

4. Vallotton, C. \& Ayoub, C. Use your words: the role of language in the development of toddlers' self-regulation. Early Child. Res. Q. 26, 169-181 (2011).

5. Council on Communications and Media. Media and young minds. Pediatrics 138, e20162591 (2016). 